\title{
NLOS Path Loss Model for Low-Height Antenna Links in High-Rise Urban Street Grid Environments
}

\author{
Juyul Lee, Myung-Don Kim, Hyun Kyu Chung, and Jinup Kim \\ Wireless Research Department, Electronics and Telecommunications Research Institute (ETRI), Daejeon 305-350, Republic of Korea \\ Correspondence should be addressed to Juyul Lee; juyul@etri.re.kr
}

Received 12 October 2014; Revised 21 January 2015; Accepted 28 January 2015

Academic Editor: Alexei Ashikhmin

Copyright (C) 2015 Juyul Lee et al. This is an open access article distributed under the Creative Commons Attribution License, which permits unrestricted use, distribution, and reproduction in any medium, provided the original work is properly cited.

\begin{abstract}
This paper presents a NLOS (non-line-of-sight) path loss model for low-height antenna links in rectangular street grids to account for typical D2D (device-to-device) communication link situations in high-rise urban outdoor environments. From wideband propagation channel measurements collected in Seoul City at $3.7 \mathrm{GHz}$, we observed distinctive power delay profile behaviors between 1-Turn and 2-Turn NLOS links: the 2-Turn NLOS has a wider delay spread. This can be explained by employing the idea that the 2-Turn NLOS has multiple propagation paths along the various street roads from TX to RX, whereas the 1-Turn NLOS has a single dominant propagation path from TX to RX. Considering this, we develop a path loss model encompassing 1-Turn and 2-Turn NLOS links with separate scattering and diffraction parameters for the first and the second corners, based on the Uniform Geometrical Theory of Diffraction. In addition, we consider the effect of building heights on path loss by incorporating an adjustable "waveguide effect" parameter; that is, higher building alleys provide better propagation environments. When compared with field measurements, the predictions are in agreement.
\end{abstract}

\section{Introduction}

Device-to-device (D2D) communication is increasingly being discussed as an emerging mobile technology and its importance is expanding as a solution to the challenge of increasing areal capacity, as well as supporting disaster situation communications $[1,2]$. From the perspective of radiowave propagation, this technology involves new propagation behaviors for mobile communications since the height of transmitters (e.g., base stations) is transitioning to near ground levels for typical outdoor D2D uses in urban areas. This leads to more interaction with objects in the close neighbor surroundings [3]. Specifically, in high-rise building environments, radiowaves from low-antenna terminals rarely propagate over-rooftop paths via diffraction but do propagate along the street roads [4]. Consequently, propagation between low-antenna terminals in high-rise urban street involves different NLOS (non-line-of-sight) behaviors with "corners" in street grids acting as the shadowing objects for NLOS conditions. Furthermore, the number of corner turns in propagation paths is an important parameter for NLOS propagation behaviors.
In this paper, we develop a NLOS path loss model for low-height terminals in high-rise urban street environments, in which corner-turning propagation paths along the street grids involve the NLOS situations. We will focus on the path loss prediction model for 1-Turn and 2-Turn NLOS links. Any two locations in regular grids can be reached via up to two corner turns. Thus, considering 1-Turn NLOS and 2Turn NLOS are enough to describe the path loss propagation in street grids, which will be later clarified. Based on the Uniform Geometrical Theory of Diffraction (UTD) [5], we derive the path loss predictions with the corner-turning effects, which include LOS-like propagation (e.g., free space path loss) behaviors along the entire propagation path from TX to RX.

By investigating the power delay profile from wideband channel measurements collected in high-rise urban streets, we observed pronounced differences in the characteristics of 1-Turn and 2-Turn NLOS links. In particular, the 2-Turn NLOS has a wider delay spread. To explain this phenomenon, we develop a path loss model for 2-Turn NLOS links separately from that for 1-Turn NLOS links. Building upon 
$\mathrm{Lu}$ et al's corner-turning path loss models [6], we derive a path loss model considering separate behaviors of 1-Turn and 2-Turn NLOS links. Additionally, we refine the free space path loss model not only for LOS propagation but also for NLOS propagation with an adjustable parameter for waveguide effects. Note that depending on the sky-line of cities, propagation behaviors vary due to the waveguide effects; for example, higher buildings in Street Canyons provide better propagation environments [7].

To validate the path loss model, we conducted measurement campaigns in Seoul City at $3.7 \mathrm{GHz}$ with a $20 \mathrm{MHz}$ bandwidth. The predictions with the path loss model are in agreement with the measured data. Although the proposed path loss model has a relatively simple form, it does provide reasonably accurate predictions for propagation behavior between low-height terminals in high-rise environments. From the perspective of the wireless system design and evaluation process for D2D communications, we expect that this new model will expedite, by simplification, the ability to estimate radiowave coverage, amount of interference, radio resource management, and so on.

Prior Work. There has been a considerable amount of prior work on path loss characteristics for urban street environments. Xia et al. [8] investigated the dependency of path loss on base station antenna height for urban NLOS propagation situations. Nagata et al. [9] found out that reflection by building walls is one of the dominant propagation mechanisms. With a ray tracing technique assuming that reflection by building walls is dominant, Erceg et al. [10] developed a path loss model in which the corner loss is characterized with a sudden drop and a steep slope change. By adding effects of diffraction, Erceg et al. [11] elaborated the path loss at corners with the Uniform Geometrical Theory of Diffraction. Molisch et al. [12] also considered a geometrical approach by introducing a local scatter model around mobile terminals. This approach was further investigated by Sun et al. [13] to combine both geometrical ray reflection and diffraction theories, which was then extended to consider a 2-turn corner loss as well. Similarly, S. Y. Tan and H. S. Tan [14] investigated the path loss behaviors at corners with diffraction and reflection mechanisms with an image concept. Berg [15] devised a recursive method to calculate path loss due to multiple diffractions at corners. Andersen et al. [16] analyzed that reflection, scattering, and diffraction are street propagation mechanism in urban building environments. All of these effects on radio propagation in urban street environments have been summarized in ITU-R (International Telecommunication Union Radiocommunication Sector) standards [17], which have been successful for planning and evaluations in "conventional" mobile communication system designs.

As an example, Figure 1 illustrates a path loss measurement data, in which both LOS and NLOS path loss data are in well agreement with the ITU-R P.1411 Street Canyon path loss model. The measurement was collected in an urban street grid area as shown in Figure 1(a), where TX was held at a stationary position $(\triangle)$ while RX was moving along the $\Delta \rightarrow \bigcirc \rightarrow \square$ route. Note that a LOS link is established from TX to RX when RX is the measurement interval $\triangle \rightarrow O$, while a NLOS link is established when RX is in $\bigcirc \rightarrow \square$ (a LOS link can be also established for some interval right after $\bigcirc$ due to wide corner areas). During the measurements, both the antennas of the transmitter and the receiver were held 1.9 meters above the ground. Details about the measurement conditions are referred to in Section 2 except that the average surrounding building height is about 11-14 meters. Since the surrounding building heights compared to the antenna heights are not so high, the ITU-R path loss model (as a representative of conventional ones) can be applied successfully.

For D2D communication scenarios, however, Nurmela et al. [18] claimed the necessity of new model considering that both the transmitter and the receiver have lowheight antennas (especially in high-rise urban areas). In this regard, Molisch and Tufvesson [3] also pointed out that D2D channels have fundamentally different nature compared to conventional cellular channels. Note also that ITU-R recognized different propagation behaviors between lowheight antenna links so that its standards were revised in September 2013 to include concepts of antenna height levels such as "below roof-top but above head level" and "at or below head level" [17].

Considering the low-height propagation characteristics, $\mathrm{Lu}$ et al. [6] recently developed a path loss model for 1Turn and 2-Turn NLOS links, based on the two-ray groundreflection model taking diffraction and scattering effects at corners into account. However, the applicability of their model is limited due to the assumptions that (1) the surrounding buildings are infinitely high, and (2) diffraction/scattering factors for 1-Turn NLOS and 2-Turn NLOS are the same. The path loss model in this paper is developed with a separate scattering/diffraction parameter for 2-Turn NLOS that can capture multiple propagation paths. Sasaki et al. [4] also considered a different nature of propagation for a different number of corner turns. However, their model is developed only for residential areas, where the average building height is less than 10 meters. In contrast to this, our model is developed for high-rise urban areas, specifically for rectilinear grid environments in Street Canyons, where average building height is far greater than 10 meters and the gap between buildings is narrower. According to the propagation path classification in [4], our model considers only "path along roads" (and not "paths between houses" or "over-roof propagation paths") since the antenna height of transmitters and receivers for D2D communications is much lower than the surrounding building height in highrise urban areas. Consequently, our model is simpler but is quite adequate for predicting propagation behaviors in urban areas. Considering the fact that D2D links are most likely established in urban areas, developing propagation models for low-height terminals in high-rise street grid environments is important.

In a similar vein, many path loss measurement results have been reported in V2V (vehicle-to-vehicle) literature as summarized in [19]. However, most of them do not investigate the environmental situations but rather focus on driving and traffic conditions. 


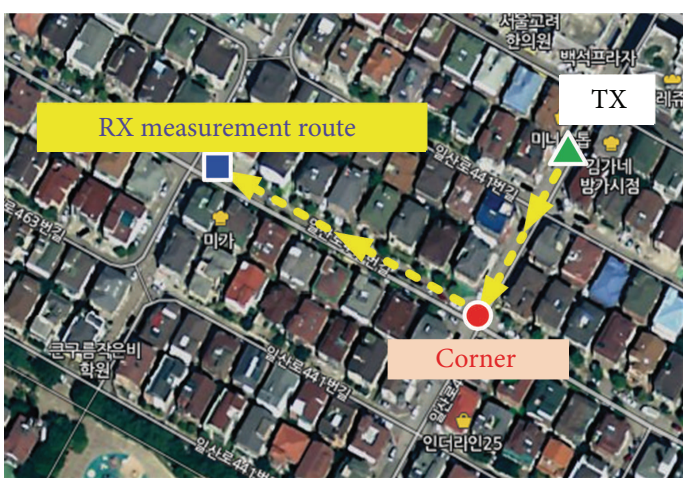

(a) Measurement environment and route

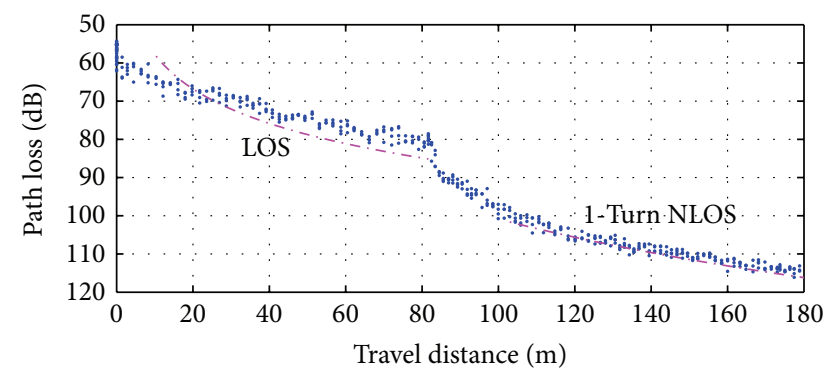

Measurement data along the route - - - ITU-R P.1411 street canyon path loss model

(b) Measurement results

FIGURE 1: Comparison with measurement data with the ITU-R P.1411 Street Canyon path loss model [17].

TABLE 1: Measurement system parameters.

\begin{tabular}{lc}
\hline Parameters & Units \\
\hline Frequency & $3.7 \mathrm{GHz}$ \\
Bandwidth & $20 \mathrm{MHz}$ \\
Chip rate & $80 \mathrm{Mcps}$ \\
PN length & $4095 \mathrm{chips}$ \\
TX power & $35 \mathrm{dBm}$ \\
RX level & $-100 \mathrm{dBm}(\mathrm{min})$ \\
\hline
\end{tabular}

\section{Measurement Overview}

2.1. Measurement Equipment Setup. The Channel Sounder system, developed at ETRI [20, 21], can measure $20 \mathrm{MHz}$ wideband multiantenna propagation characteristics at a carrier frequency $3.7 \mathrm{GHz}$. The transmitter part (TX) of the sounder transmits pseudonoise (PN) sequences in a $20 \mathrm{MHz}$ bandwidth and then the receiver part (RX) samples MIMO channels in a TD (time division) snapshot fashion, that is, multiple receiver antennas sequentially receive sounding PN signals from multiple transmit antennas. The Channel Sounder system parameters are provided in Table 1.

Oversampling by a factor of $4, \mathrm{RX}$ calculates the channel impulse response at a rate of $80 \mathrm{MHz}$, while storing the GPS location information for distance measures. Path loss is then calculated by using the sampled received power, compensating for AGC (Automatic Gain Control) levels in $\mathrm{RX}$, cable loss, antenna gain, and so forth.

To measure the path loss between low-height antenna terminals in street grid environments, we equipped TX and RX in vehicles, as illustrated in Figure 2, where the antennas were installed on the rooftop of the vehicles (1.9 meters above the ground). To improve measurement data quality, we installed four antennas in RX, in which the antenna separations were 10 wavelengths to achieve independent fading. The received power is calculated by averaging received powers from the four antennas.

2.2. Measurement Environments. The measurement campaigns were conducted at Yeouido district in Seoul City,

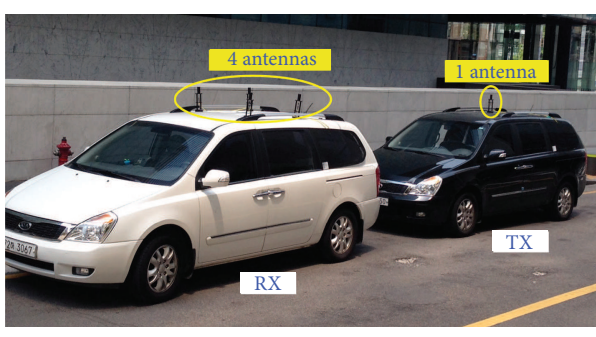

FIGURE 2: Equipment installation on vehicles where 1 omni-directional antenna in TX and 4 omni-directional antennas (separated by 10 wavelengths) in RX are installed on the rooftop.

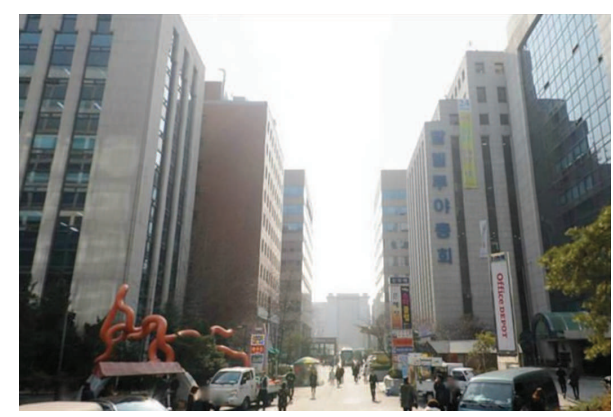

FIGURE 3: Measurement environments: urban street with $35-45 \mathrm{~m}$ high buildings and $30 \mathrm{~m}$ wide street.

which is a commercial area with 10-15 story buildings (35$45 \mathrm{~m}$ height) and $30 \mathrm{~m}$ wide streets (distance measured between buildings included the sidewalks) as illustrated in Figure 3. Note that this area has regular street grids and can be categorized with an urban high-rise environment per ITU$\mathrm{R}$ standard [17].

We carried out the measurement campaigns with the route scenarios as shown in Figure 4, which are designed to include LOS, 1-Turn NLOS, and 2-Turn NLOS situations. Scenario 1 in Figure $4(\mathrm{a})$ is a case that the straight line distance from TX to RX increases monotonically as the RX movement travel distance increases. Scenario 2 is a case that $\mathrm{RX}$ is circularly traveled so that the 2-Turn NLOS 


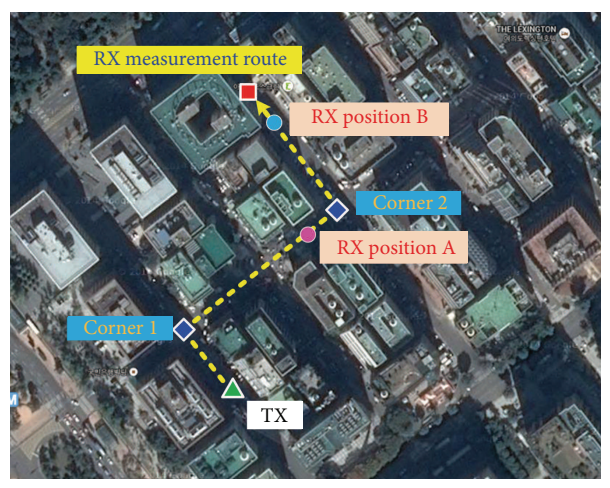

(a) Scenario 1

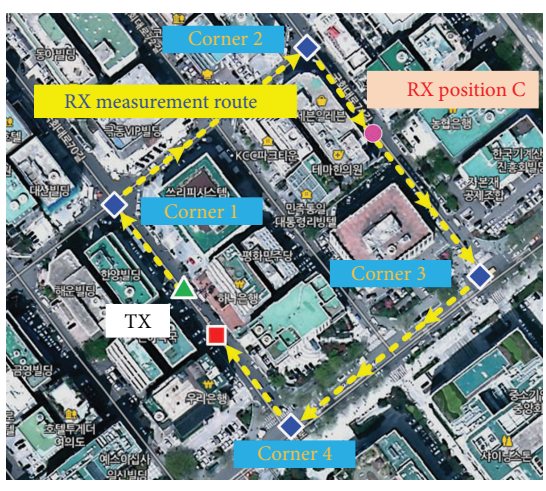

(b) Scenario 2

FIGURE 4: Measurement route in Seoul City: TX was held at a stationary position while RX moved along the dotted route path.

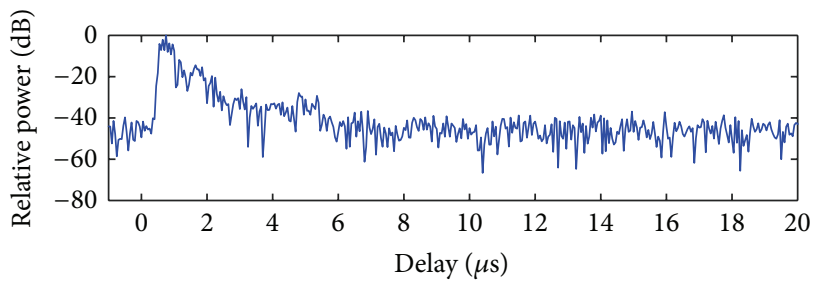

(a) Normalized PDP measured at Position A (1-Turn NLoS)

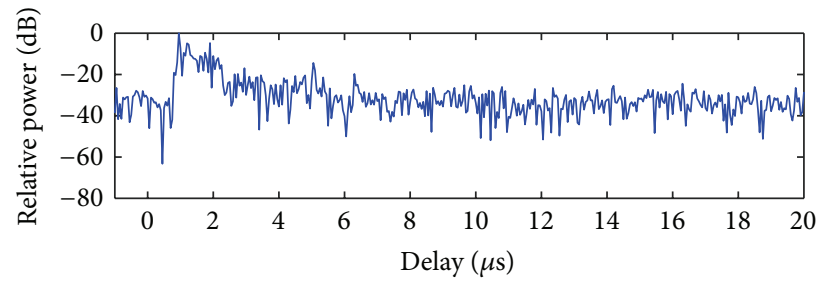

(b) Normalized PDP measured at Position B (2-Turn NLoS)

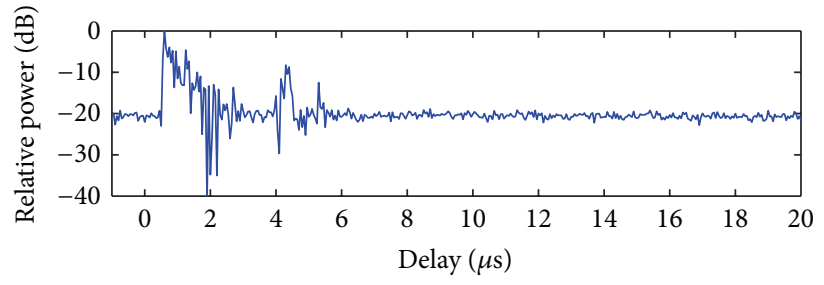

(c) Normalized PDP measured at Position C (2-Turn NLoS)

Figure 5: Power delay profile (PDP) measurements between TX and RX positions in Figure 4.

interval (from corner 2 to corner 3 in Figure 4(b)) can have stationary path loss behaviors, because the backward propagation distance decreases as the RX travel distance increases. The forward and the backward propagation paths mean that the former is the propagation path from TX to RX along the RX movement path and the latter is along the other side path. To measure path loss characteristics, we fixed TX at a stationary location marked $\triangle$ in Figures 4 (a) and 4(b) and moved RX (under $10 \mathrm{~km} / \mathrm{h}$ ) along the dotted measurement routes marked $\Delta \rightarrow \diamond \rightarrow \cdots \rightarrow \diamond \rightarrow \square$ in Figures 4(a) and 4(b). The campaigns were conducted during daytime (outside of rush/lunch hours) when few people were on sidewalks and vehicle traffic was running at under $30 \mathrm{~km} / \mathrm{h}$.

\section{1-Turn NLOS versus 2-Turn NLOS}

This section investigates radiowave propagation characteristics of 1-Turn NLOS and 2-Turn NLOS in street grids with wideband channel measurements. In terms of propagation, 2-Turn NLOS is different from 1-Turn NLOS not only due to the number of corner turns but also due to the number of propagation routes when the PDP measurements are observed. We also point out the importance of 2-Turn NLOS situations in street grid environments by examining the occurrences of 1-Turn NLOS and 2-Turn NLOS.

\subsection{Power Delay Profiles of 1-Turn NLOS and 2-Turn NLOS.} Based on measurement data, we examine power delay profile characteristics of 1-Turn NLOS and 2-Turn NLOS situations in Figure 4. Figure 5 shows normalized power delay profiles (PDP), relative to the peak power, measured at RX positions $A$ and B marked in Figure 4(a) and position C in Figure 4(b). From the PDP of the 1-Turn NLOS in Figure 5(a), we can observe that the delay spread is relatively short (peak delay is spread between $0.5 \mu \mathrm{s}$ and $1 \mu \mathrm{s}$ ). For the 2-Turn NLOS of Scenario 1 in Figure 5(b), power delay is more widely spread (between $0.8 \mu \mathrm{s}$ and $2.3 \mu \mathrm{s}$ ). Similar phenomenon is observed for Scenario 2 in Figure 5(c). Moreover, the 2-Turn NLOS of Scenario 2 has an additional multipath components in PDP between $4 \mu \mathrm{s}$ and $4.5 \mu \mathrm{s}$, which is due to the backward propagation route. In the power calculation of path loss, all the received power should be added together. Notice that such 


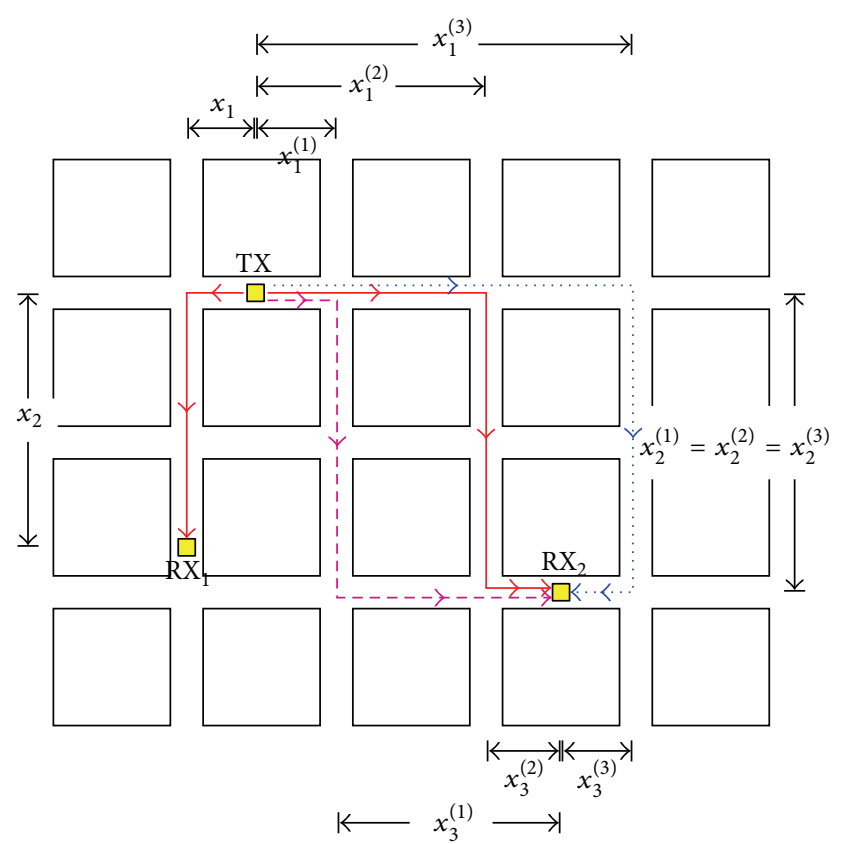

Figure 6: 1-Turn (between TX and $\mathrm{RX}_{1}$ ) and 2-Turn (between TX and $\mathrm{RX}_{2}$ ) NLOS examples: the 1-Turn NLOS has a single propagation path while the 2-Turn NLOS has 3 comparable propagation paths.

multiple 2-Turn NLOS components have been overlooked in the literature.

As explained, the delay spread difference is due to the number of propagation paths from TX to RX. To illustrate different number of propagation paths, Figure 6 shows a typical situation for 1-Turn NLOS (from TX to $\mathrm{RX}_{1}$ ) and 2-Turn NLOS (from TX to $\mathrm{RX}_{2}$ ). For the 1-Turn NLOS link from $\mathrm{TX}$ to $\mathrm{RX}_{1}$, only one single propagation path, from the perspective of propagation, is dominant among many possible propagation paths. The contribution of the radiowaves through other paths is relatively minuscule and can be ignored in the received power calculation. For the 2Turn NLOS link from TX to $\mathrm{RX}_{2}$, however, there are three dominant propagation paths; that is, radiowaves propagation through the three paths has comparable attenuation levels. Thus, when characterizing these NLOS path losses in street grids, an additional corner turn does not simply mean an additional propagation loss at a corner but should also be considered with the changes of propagation paths, including the number of paths as well as the number of corners.

3.2. Occurrences of 1-Turn NLOS and 2-Turn NLOS. In rectangular street grids, we now investigate the occurrences of 1-Turn NLOS and 2-Turn NLOS situations, depending on locations of TX and RX to emphasize the importance of 2Turn NLOS situations. Without loss of generality, we fix the location of TX and consider link types with respect to the location of RX. When TX is at a corner, no 2-Turn NLOS link is established; that is, all the links can be described with either LOS or 1-Turn NLOS, no matter where RX is located. In this respect, 2-Turn NLOS situations do not need to be considered for conventional cellular networks in very high-rise urban

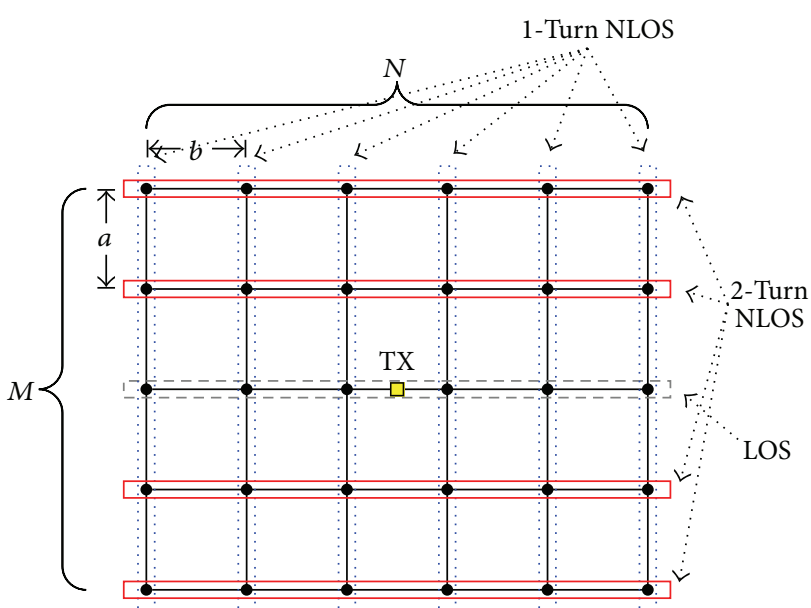

FIgURE 7: LOS, 1-Turn NLOS, and 2-Turn NLOS situations in an $M \times$ $N$ rectangular street grid: black dots and edges denote corners and streets in the street grid.

areas if base stations are located at corner areas. In very high-rise areas (e.g., Manhattan in New York), base station antennas are installed below rooftops since the rooftops are too high. When TX is not in a corner area, however, 2-Turn NLOS situations exist. Since TX is a mobile terminal in D2D links, TX can be placed at any location along the street (and, more likely, not placed at a corner).

To illustrate the situations of 1-Turn and 2-Turn NLOS, when TX is not at a corner, let us consider an $M \times N$ rectangular grid as shown in Figure 7 , where $M(\geq 2)$ and $N(\geq 2)$ are the number of corner points in horizontal and vertical axis, respectively. In the figure, black dots and edges denote corners and streets in the street grid, respectively. LOS situations occur when RX is located on the same street as TX, 1-Turn NLOS situations occur when RX is located in the blue-dotted areas in Figure 7, and 2-Turn NLOS situations occur when RX is located in the red-line areas. If we assume that corners in the grid are equally spaced by distances $a$ (in horizontal axis) and $b$ (in vertical axis), the total length of LOS intervals is $l_{\mathrm{LOS}}=b(N-1)$. The total length of the streets in the category of 1-Turn NLOS is $l_{1 \text {-Turn }}=a(M-1) N$, while that in the 2-Turn NLOS category is $l_{2 \text {-Turn }}=b(M-1)(N-1)$. The fraction of the total street length $l_{\text {total }}$ in the 2-Turn NLOS category is given by

$$
\frac{l_{2 \text {-Turn }}}{l_{\text {total }}}=\frac{b(M-1)(N-1)}{a(M-1) N+b M(N-1)},
$$

where $l_{\text {total }}$ is the total length of the $M \times N$ grid. If we further assume that $M=N$ and $a=b$, then almost $50 \%$ of the grid corresponds to 2-Turn NLOS at large $M$ since $l_{2 \text {-Turn }} / l_{\text {total }}=$ $0.5-1 /(2 M)$. Consequently, developing a separate path loss model for 2-Turn NLOS links is important. Additionally, considering more than two corner turns is not required for regular street grids. 


\section{Path Loss Model}

In this section we develop a path loss model for 1-Turn NLOS and 2-Turn NLOS situations involving low-height antenna terminals in rectangular street grid environments. Based on the Uniform Geometrical Theory of Diffraction [5], we quantify the path loss ratio relative to the LOS path loss due to corner turns. In doing so, we exploit the ITU$\mathrm{R}$ path loss model [17] for the LOS propagation, which is actually a de facto standard when the model is applicable (so far the ITU-R model does not include a site-specific NLOS path loss prediction model between low-height terminals in urban street environments [17]). Helpfully, the ITU-R LOS path loss model has some inherent adjustability since the model is given by an upper and a lower bound, which we exploit to parameterize waveguide effects. In this way, we can incorporate both corner-turning effects and waveguide effects on the path loss model. To verify the proposed path loss model, we compare the resulting calculation to field measurement data.

4.1. Path Loss Model Derivation. We begin the path loss model derivation with 1-Turn NLOS situations. As illustrated in Figure 6, one corner is located in the propagation path from TX to $\mathrm{RX}_{1}$. The distance from TX to the corner and the distance from the corner to $\mathrm{RX}_{1}$ are denoted by $x_{1}$ and $x_{2}$, respectively. From the Uniform Geometrical Theory of Diffraction [22, Chapter 5] (originally in [5]), the path loss due to diffraction at an edge of a corner is given by [22, Eq. (5-62)]

$$
\mathrm{PL}_{1-\text { Turn }}^{\text {edge }}=\left(\frac{4 \pi}{\lambda}\right)^{2} \frac{\cos ^{2} \psi}{D_{1}^{2}} x_{1} x_{2}\left(x_{1}+x_{2}\right),
$$

where $\lambda$ is the wavelength of the operating center frequency, $D_{1}$ is the diffraction factor at the associated edge, and $\psi$ is the viewing angle from the transmiting antenna to the receiver antenna, which is dependent on the heights of antennas. This viewing angle factor is to account for the oblique incidence to the corner edges due to the antenna height differences. According to [22], (2) is valid when the antennas are isotropic in both azimuthal and elevation planes. Although we used omnidirectional dipole antennas, that is, isotropic in the azimuthal plane but nonisotropic in the elevation direction, we assume that (2) is approximately valid with the dipole antennas. Considering that similar phenomena happen at the other edges and that $x_{1}$ and $x_{2}$ for all four edges are approximately the same, the path loss for 1-Turn NLOS can be written as

$$
\mathrm{PL}_{1-\mathrm{Turn}}=\left(\frac{4 \pi}{\lambda}\right)^{2} \frac{\cos ^{2} \psi}{S_{1}^{2}} x_{1} x_{2}\left(x_{1}+x_{2}\right),
$$

where $S_{1}$ is an integrating factor, combining diffraction and scattering phenomena at all the four edges in the associated corner.

By dividing the free space path loss at $x_{1}+x_{2}$ (the travel distance between the transmitter and the receiver is $x_{1}+x_{2}$ ), the relative path loss of 1-Turn to the free space path loss is given by

$$
\frac{\mathrm{PL}_{1-\text { Turn }}}{\mathrm{PL}_{\text {free-space }}}=\frac{\cos ^{2} \psi}{S_{1}^{2}} \frac{x_{1} x_{2}}{x_{1}+x_{2}} .
$$

Here, we exploit the ITU-R LOS path loss model for the free space loss, which is given by [17]

$$
\mathrm{PL}_{\mathrm{LOS}}= \begin{cases}10^{\alpha_{\mathrm{dg}} / 10}\left(\frac{\lambda^{2}}{8 \pi h_{\mathrm{TX}} h_{\mathrm{RX}}}\right)^{2}\left(\frac{d}{R_{\mathrm{bp}}}\right)^{2.5}, & \text { if } d \leq R_{\mathrm{bp}}, \\ 10^{\alpha_{\mathrm{ds}} / 10}\left(\frac{\lambda^{2}}{8 \pi h_{\mathrm{TX}} h_{\mathrm{RX}}}\right)^{2}\left(\frac{d}{R_{\mathrm{bp}}}\right)^{4}, & \text { if } d>R_{\mathrm{bp}},\end{cases}
$$

where $R_{\mathrm{bp}}$ is the break point distance calculated by $4 h_{\mathrm{TX}} h_{\mathrm{RX}} / \lambda$ and $h_{\mathrm{TX}}$ and $h_{\mathrm{RX}}$ are the antenna heights of TX and RX, respectively. Distance $d$ should be calculated with the travel distance between the transmitter and the receiver; that is, $d=x_{1}+x_{2}$. Parameter $\alpha_{\mathrm{dB}}$ is used to adjust the path loss level (in $\mathrm{dB}$ scale) to account for waveguide effects attributed by deep alleys in Street Canyons. In the ITU-R LOS model [17], $\alpha_{\mathrm{dB}}=0$ corresponds to the lower bound of the path loss and $\alpha_{\mathrm{dB}}=20$ corresponds to the upper bound; that is, $0 \leq \alpha_{\mathrm{dB}} \leq 20$. As reported in [7], since higher building alleys provide better propagation environments, $\alpha_{\mathrm{dB}}$ will be smaller for higher building environments. Consequently, the path loss for the 1-Turn NLOS situation is given by

$$
\mathrm{PL}_{1-\text { Turn }}=\mathrm{PL}_{\mathrm{LOS}} \frac{\cos ^{2} \psi}{S_{1}^{2}} \frac{x_{1} x_{2}}{x_{1}+x_{2}} .
$$

Since this path loss formula has a relatively simple form, with few input environmental parameters, the computation time will be short enough to permit real-time applications. Moreover, note that the formula is symmetric with respect to $x_{1}$ and $x_{2}$, and therefore channel reciprocity holds. The channel reciprocity means that the propagation characteristic from a transmitter to a receiver is identical to that from the receiver to the transmitter; that is, the channel propagation characteristic is invariant when swapping the location of the transmitter and the location of the receiver. Note also that this formula is identical to the 1-Turn NLOS path loss model in [6] if $\psi=0$ and $\mathrm{PL}_{\mathrm{LOS}}$ is replaced by the two-ray ground reflection model. In outdoor urban environments, $\psi=0$ may be typical for D2D links since the heights of TX and $\mathrm{RX}$ are likely to be the same. However, $\mathrm{PL}_{\mathrm{LOS}}$ term will be significantly varied due to different amount of waveguide effects, depending on the relative building heights to the antenna heights, which are not considered in [6].

We now develop a path loss model for 2-Turn NLOS situations. As illustrated in Figure 6, at least two corner turns are required to reach from TX to $\mathrm{RX}_{2}$ regardless of propagation routes. As discussed in the previous section, 2-Turn NLOS links can have various propagation routes depending on the locations of the transmitter and the receiver, whereas 1-Turn NLOS links have, for the most part, a single dominant propagation path. For example, the 2-Turn NLOS link in 
Figure 6 (i.e., from $\mathrm{TX}$ to $\mathrm{RX}_{2}$ shown in the figure) has 3 propagation route paths, in which the 3 paths are likely to have similar attenuation levels. In this case of multiple propagation travel paths, radio waves from all the paths are independently added at the receiver, and thus the received power is calculated by summing all the received powers from all the travel paths. Therefore, the received power gain in terms of path loss is calculated by

$$
\frac{1}{\mathrm{PL}_{2-\text { Turn }}}=\sum_{n} \frac{1}{\mathrm{PL}_{2 \text {-Turn }}^{\text {(nth path) }}\left(x_{1}^{\text {(nth path) }}, x_{2}^{\text {(nth path) }}, x_{3}^{\text {(nth path) }}\right)},
$$

where $\mathrm{PL}_{2 \text {-Turn }}^{\text {(nth path) }}$ is the path loss of the $n$th 2-Turn travel route path. The $n$th 2 -Turn travel route path comprises the following: the distance from the transmitter to the first corner, the first corner to the second corner, and the second corner to the receiver are denoted by $x_{1}^{\text {(nth path) }}, x_{2}^{\text {(nth path) }}$, and $x_{3}^{\text {(nth path) }}$, respectively, and are illustrated in Figure 6.

As was done for the 1-Turn NLOS situations, the path loss of the $n$th 2-Turn travel route path can be also obtained with the Uniform Geometrical Theory of Diffraction as [22, Eq. $(5-67)]$

$$
\begin{aligned}
\mathrm{PL}_{2-\text { Turn }}^{\text {(nth path })}= & \left(\frac{4 \pi}{\lambda}\right)^{2} \frac{\cos ^{4} \psi}{S_{1}^{2} S_{2}^{2}} x_{1}^{(n \text {th path })} x_{2}^{(n \text {th path })} x_{3}^{(n \text {th path })} \\
& \cdot\left(x_{1}^{(n \text {th path })}+x_{2}^{(\text {nth path) }}+x_{3}^{(n \text {th path })}\right),
\end{aligned}
$$

where $S_{1}$ and $S_{2}$ are the integrated scattering and diffraction parameters for the first corner turns and the second corner turns, respectively. We can reuse $S_{1}$ obtained from the 1-Turn NLOS situation as in (6). For $S_{2}$, care should be taken in that this parameter is concerned with all the second corner turns for all possible traveling paths between the transmitter and the receiver (not for a single propagation path); for example, all the three propagation paths in Figure 6 should be considered together to determine $S_{2}$. Consequently, $S_{1}$ is determined by a single corner in a single traveling path, whereas $S_{2}$ is determined by all the second corners in multiple comparable traveling route paths. To reiterate, this is because the shortest single traveling route path is the dominant propagation path for 1-Turn NLOS while multiple comparable traveling route paths are dominant together for 2-Turn NLOS. In this way, we determine corner effect parameters $S_{1}$ and $S_{2}$, separately ( $\mathrm{Lu}$ et al. assumed that $S_{1}=S_{2}$ in their derivation [6]).

By replacing the free space path loss in (8) at $d=$ $x_{1}^{\text {(nth path) }}+x_{2}^{\text {(nth path) }}+x_{3}^{\text {(nth path) }}$ with the ITU-R LOS path loss, we have

$$
\begin{aligned}
& \mathrm{PL}_{2 \text {-Turn }}^{\text {(nth path) }} \\
& \quad=\mathrm{PL}_{\mathrm{LOS}} \frac{\cos ^{4} \psi}{S_{1}^{2} S_{2}^{2}} \frac{x_{1}^{(n \text {th path) }} x_{2}^{\text {(nth path) }} x_{3}^{\text {(nth path) }}}{x_{1}^{\text {(nth path) }}+x_{2}^{\text {(nth path) }}+x_{3}^{(\text {nth path) }}},
\end{aligned}
$$

where $\mathrm{PL}_{\mathrm{LOS}}$ is defined in (5) and plays a role of compensating for waveguide effects due to the deep alleys in Street Canyons.

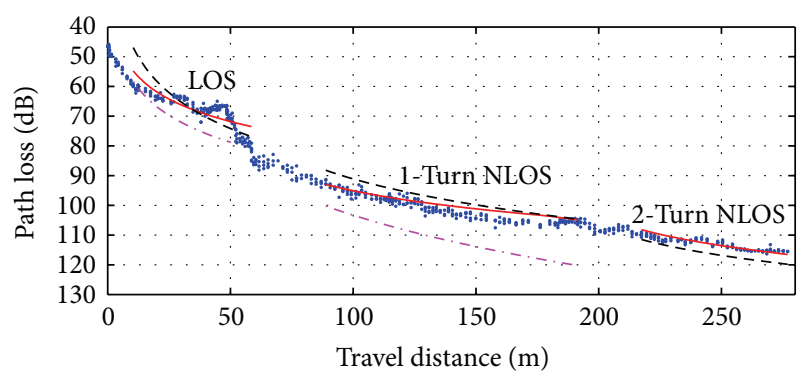

$$
\begin{array}{ll} 
& \text { Measurement data from scenario } 1 \\
\text { _ } & \text { Proposed path loss model } \\
\text { - - - } & \text { Lu et al. path loss model } \\
\text { - - - } & \text { ITU-R P.1411 street canyon path loss model }
\end{array}
$$

FIGURE 8: Comparison of the proposed path loss model (fitting to the measurement data), Lu et al. model in [6], and the ITU-R Street Canyon model [17] for Scenario 1 in Figure 4(a).

Notice that both (6) for 1-Turn NLOS and (9) for 2-Turn NLOS include $\mathrm{PL}_{\mathrm{LOS}}$ calculated with the total travel distance from the transmitter and the receiver. This can be understood by employing the idea that the entire propagation path has LOS-like propagation behaviors since the radio waves propagate street roads from a low-height TX to a low-height RX in high-rise building alleys. Then, corner-turning effects are considered by the other terms in (6) and (9).

Although the path loss calculations for 2-Turn NLOS in (7) and (9) seem to be complicated compared to those for 1-Turn NLOS, they are, actually, relatively simple arithmetic computations. As a result, together with the 1-Turn model, this path loss model can be implemented with low complexity. Note that $\mathrm{PL}_{2 \text {-Turn }}^{(\text {nth path) }}$ in (9) is symmetric with respect to $x_{1}^{\text {(nth path) }}, x_{2}^{\text {(nth path) }}$, and $x_{3}^{\text {(nth path) }}$, and therefore interchanging the locations of the transmitter and the receiver does not affect $\mathrm{PL}_{2 \text {-Turn }}$ in (7). Consequently, the path loss model for both 1-Turn NLOS and 2-Turn NLOS has channel reciprocity.

4.2. Verification with Measurement Data. To validate the proposed path loss model, we compare it with the field measurement data as well as the conventional path loss models. Since the proposed path loss model considers only NLOS situations, we use the formula in (5) to predict the path loss for LOS component parts. Details about the field measurement data such as measurement equipment setup and measurement campaigns are referred to in Section 2. As a representative of conventional path loss model, ITUR P.1411 Street Canyon standard model [17] is chosen for comparison. As discussed in Figure 1, the ITU-R model is in well agreement with relatively mid-rise (compared to the antenna heights) urban street grid environments.

Figure 8 shows the comparison of the proposed path loss model with measurement data, which were collected following Scenario 1 in Figure 4(a). Scenario 1 includes link situations for LOS (the travel distance from 0 to $60 \mathrm{~m}$, marked $\Delta \rightarrow \diamond$ (corner 1) in Figure 4(a)), 1-Turn NLOS (the travel distance from $85 \mathrm{~m}$ to $190 \mathrm{~m}$, marked $\diamond($ corner 1$) \rightarrow$ $\diamond($ corner 2)), and 2-Turn NLOS (the travel distance from 


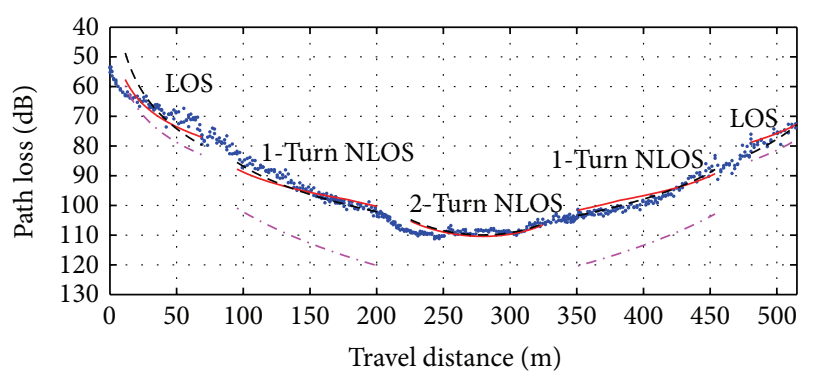

$$
\begin{aligned}
& \text { Measurement data from scenario } 1 \\
& \text { _ Proposed path loss model } \\
& \text { - - - Lu et al. path loss model } \\
& \text { - - - ITU-R P.1411 street canyon path loss model }
\end{aligned}
$$

FIGURE 9: Comparison of the proposed path loss model (fitting to the measurement data), Lu et al. model in [6], and the ITU-R Street Canyon model [17] for Scenario 2 in Figure 4(b).

$220 \mathrm{~m}$ to $270 \mathrm{~m}$, marked $\diamond($ corner 2$) \rightarrow \square$ ). The travel distance denotes the RX movement distance along the measurement route. Since the initial RX position is the same with the TX position, TX is located at zero travel distance. Recall that Scenario 1 is designed such that the distance between TX and RX increases as the travel distance increases. As can be seen in Figure 8, the path loss predictions are in well agreement with the measurement data. Compared with $\mathrm{Lu}$ et al's model, the proposed model has better fitting. To be specific, RMSE (root-mean-square-error) for the proposed model and Lu et al.s model are calculated by $2.44 \mathrm{~dB}$ and $4.20 \mathrm{~dB}$, respectively, where the RMSE is calculated by

$$
\mathrm{RMSE}=\sqrt{\frac{1}{L} \sum_{l=1}^{L}\left(\mathrm{PL}_{\text {measurement }}(l)-\mathrm{PL}_{\mathrm{fit}}(l)\right)^{2}},
$$

where $l$ is the measurement sample index and $L$ is the total number of samples. Since GPS receivers are installed in the measurement equipment, we are able to obtain the distance information to calculate the path loss prediction at every sample index. Note that the conventional model (ITUR P.1411 Street Canyon model [17]) provides considerable overestimation for both LOS and 1-Turn NLOS parts. The ITU-R model has yet to consider 2-Turn NLOS situations.

Figure 9 shows the comparison results for Scenario 2 depicted in Figure 4(b). The RX unit in Scenario 2 was moved along a circulation route:

$$
\begin{aligned}
\Delta & \stackrel{\text { LOS }}{\longrightarrow} \diamond(\text { corner } 1) \stackrel{1 \text {-Turn }}{\longrightarrow} \diamond(\text { corner } 2) \\
& \stackrel{\text { 2-Turn }}{\longrightarrow} \diamond(\text { corner } 3) \stackrel{1 \text {-Turn }}{\longrightarrow} \diamond(\text { corner } 4) \stackrel{\text { LOS }}{\longrightarrow} \square
\end{aligned}
$$

while TX was fixed at $\triangle$ to include LOS, 1-Turn NLOS, and 2-Turn NLOS situations as marked in the above diagram. Compared with the measurement data shown in the figure, both the proposed path loss model and the Lu et al's model are are in well agreement. In particular, both models provide relatively accurate path loss predictions for the 2-Turn NLOS interval (between corner 2 and corner 3 ), in which radiowaves from TX to RX propagate not only through the forward direction (with respect to the RX movement direction) but also through the backward direction. In terms of the RMSE performance measure, the error of the proposed model is $2.73 \mathrm{~dB}$ while that of Lu et al.s model is $3.65 \mathrm{~dB}$. However, the conventional ITU-R Street Canyon model provides significant amount of overestimation for LOS and 1-Turn NLOS.

Thus far, we have compared path loss models with field measurement data. We have seen that the conventional ITUR Street Canyon model provides overestimated path loss predictions for LOS and 1-Turn NLOS situations, owing to the fact that low-height terminal link conditions in highrise Street Canyons have yet to be considered properly. In addition, the ITU-R model does not provide predictions for 2-Turn NLOS situation. We have also shown that the predictions with the proposed model have smaller RMSE than those with the Lu et al.s model, which is due to the fact that the proposed model has a waveguide effect consideration term and individual corner effect factors for the first corner turn and the second corner turn.

\section{Conclusion}

For low-height antennas link terminals in outdoor urban street environments, we developed a path loss model with the Uniform Geometrical Theory of Diffraction, especially for NLOS situations due to corner turns in street grids. The proposed model provides path loss predictions for 1-Turn and 2-Turn NLOS situations, while accounting for waveguide effects due to high-rise building alleys. In particular, we employed a NLOS extension with the ITU-R LOS path loss model, which eventually provides a reasonable explanation about propagation along the street roads between low-height antenna terminals in street grid environments. Although the proposed path loss model is a simple formula, with arithmetic calculations and few environmental parameter inputs, it can provide reasonably accurate predictions.

\section{Conflict of Interests}

The authors declare that there is no conflict of interests regarding the publication of this paper.

\section{Acknowledgments}

The authors would like to thank Professor Henry L. Bertoni and Dr. Jonathan S. Lu for helpful discussions on 1-Turn and 2-Turn NLOS links, as well as anonymous reviewers. This work was supported by the IT R\&D programs of MSIP (Ministry of Science, ICT, and Future Planning), Korea [15ZI1110, Research on Advanced Technologies of Access Network for Traffic Capacity Enhancement].

\section{References}

[1] 3GPP TR 22.803, "Feasibility study for Proximity Services (ProSe)," March 2013.

[2] M. Tehrani, M. Uysal, and H. Yanikomeroglu, "Device-todevice communication in $5 \mathrm{G}$ cellular networks: challenges, 
solutions, and future directions," IEEE Communications Magazine, vol. 52, no. 5, pp. 86-92, 2014.

[3] A. F. Molisch and F. Tufvesson, "Propagation channel models for next-generation wireless communications systems," IEICE Transactions on Communications, vol. E97.B, no. 10, pp. 20222034, 2014.

[4] M. Sasaki, W. Yamada, N. Kita, and T. Sugiyama, "Path loss model with low antenna height for microwave bands in residential areas," IEICE Transactions on Communications, vol. 96, no. 7, pp. 1930-1944, 2013.

[5] D. A. McNamara, C. W. Pistorius, and J. A. Malherbe, Introduction to the Uniform Geometrical Theory of Diffraction, Artech House, Boston, Mass, USA, 1990.

[6] J. S. Lu, H. L. Bertoni, K. A. Remley, W. F. Young, and J. Ladbury, "Site-specific models of the received power for radio communication in urban street canyons," IEEE Transactions on Antennas and Propagation, vol. 62, no. 4, pp. 2192-2200, 2014.

[7] J. Lee, M. D. Kim, J. Kim, and H. K. Chung, "Path loss measurements for low-antenna links in urban street environments," in Proceedings of the IEEE Antennas and Propagation Society International Symposium (APSURSI '14), pp. 957-958, Memphis, Tenn, USA, July 2014.

[8] H. H. Xia, H. L. Bertoni, L. R. Maciel, A. Lindsay-Stewart, and R. Rowe, "Microcellular propagation characteristics for personal communications in urban and suburban environments," IEEE Transactions on Vehicular Technology, vol. 43, no. 3, pp. 743-752, 1994.

[9] Y. Nagata, Y. Furuya, E. Moriyama, M. Mizuno, I. Kamiya, and S. Hattori, "Measurement and modeling of $2 \mathrm{GHz}$ band outof-sight radio propagation characteristics under microcellular environments," in Proceedings of the IEEE International Symposium on Personal, Indoor and Mobile Radio Communications., pp. 341-346, 1991.

[10] V. Erceg, S. Ghassemzadeh, M. Taylor, D. Li, and D. L. Schilling, "Urban/suburban out-of-sight propagation modeling," IEEE Communications Magazine, vol. 30, no. 6, pp. 56-61, 1992.

[11] V. Erceg, A. J. Rustako Jr., and R. S. Roman, "Diffraction around corners and its effects on the microcell coverage area in urban and suburban environments at $900 \mathrm{MHz}, 2 \mathrm{GHz}$, and $6 \mathrm{GHz}$," IEEE Transactions on Vehicular Technology, vol. 43, no. 3, pp. 762-766, 1994.

[12] A. F. Molisch, A. Kuchar, J. Laurila, K. Hugl, and E. Bonek, "Efficient implementation of a Geometry-based directional model for mobile radio channels," in Proceedings of the Fall. IEEE VTS 50th Vehicular Technology Conference (VTC '99), vol. 3, pp. 1449-1453, September 1999.

[13] Q. Sun, S. Y. Tan, and K. C. Teh, "Analytical formulae for path loss prediction in urban street grid microcellular environments," IEEE Transactions on Vehicular Technology, vol. 54, no. 4, pp. 1251-1258, 2005.

[14] S. Y. Tan and H. S. Tan, "Theory for propagation path-loss characteristics in a city-street grid," IEEE Transactions on Electromagnetic Compatibility, vol. 37, no. 3, pp. 333-342, 1995.

[15] J.-E. Berg, "A recursive method for street microcell path loss calculations," in Proceedings of the 6th IEEE International Symposium on Personal, Indoor and Mobile Radio Communications (PIMRC '95), pp. 140-143, September 1995.

[16] J. B. Andersen, T. S. Rappaport, and S. Yoshida, "Propagation measurements and models for wireless communications channels," IEEE Communications Magazine, vol. 33, no. 1, pp. 42-49, 1995.
[17] ITU, "Propagation data and prediction methods for the planning of short-range outdoor radiocommunication systems and radio local area networks in the frequency range $300 \mathrm{MHz}$ to 100 GHz," Recommendation ITU-R P.1411-7, 2013.

[18] V. Nurmela, T. Jämsä, P. Kyösti, V. Hovinen, and J. Medbo, "Channel modelling for device-to-device scenarios," Tech. Rep. COST IC1004, 2013.

[19] A. F. Molisch, F. Tufvesson, J. Karedal, and C. F. Mecklenbräuker, "A survey on vehicle-to-vehicle propagation channels," IEEE Wireless Communications, vol. 16, no. 6, pp. 12-22, 2009.

[20] J.-J. Park, M.-D. Kim, H.-K. Kwon, H. K. Chung, X. Yin, and $\mathrm{Y}$. Fu, "Models of a multilink channel in cooperative communication environments," ETRI Journal, vol. 34, no. 6, pp. 858-868, 2012.

[21] M. D. Kim, J. J. Park, H. K. Kwon, and H. K. Chung, "Performance evaluation of wideband MIMO relay channel sounder for $3.7 \mathrm{GHz}$," in Proceedings of the IEEE Vehicular Technology Society Asia Pacific Wireless Communications Symposium (APWCS '11), August 2011.

[22] H. L. Bertoni, Radio Propagation for Modern Wireless Systems, Prentice Hall, Upper Saddle River, NJ, USA, 2000. 

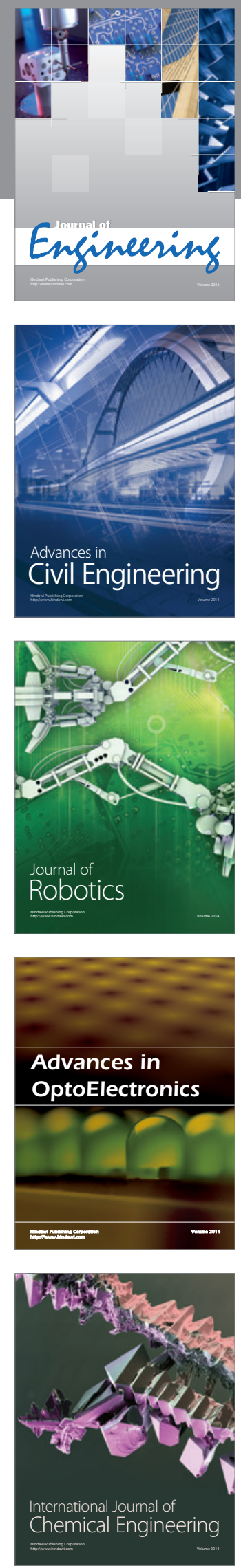

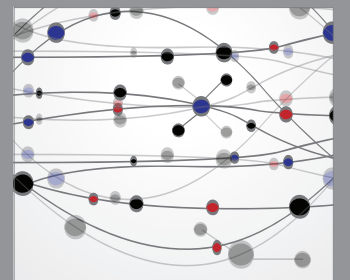

The Scientific World Journal
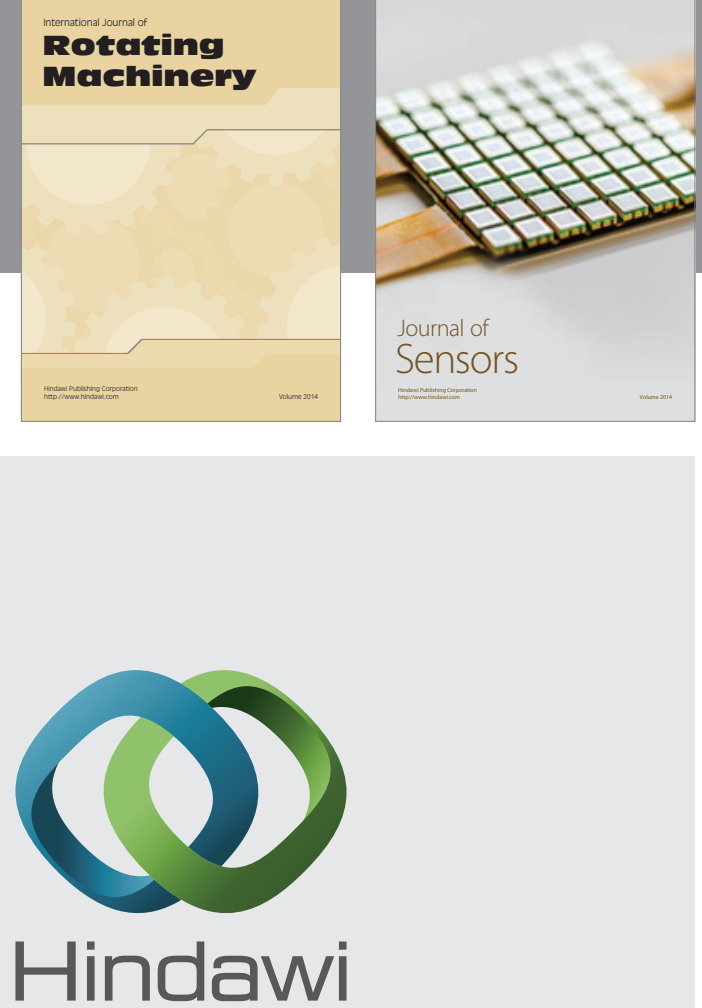

Submit your manuscripts at http://www.hindawi.com
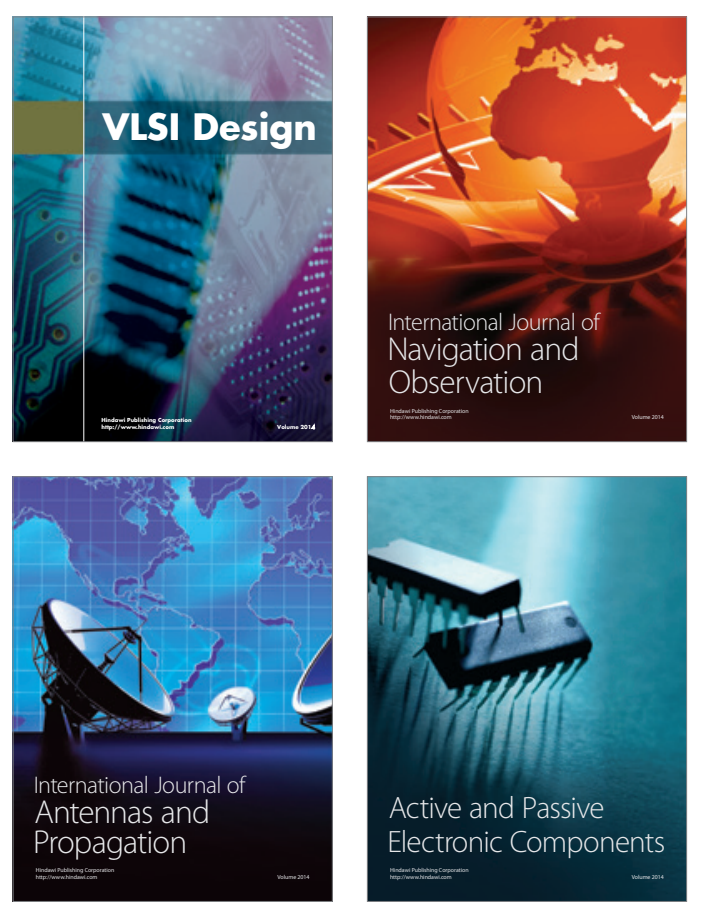
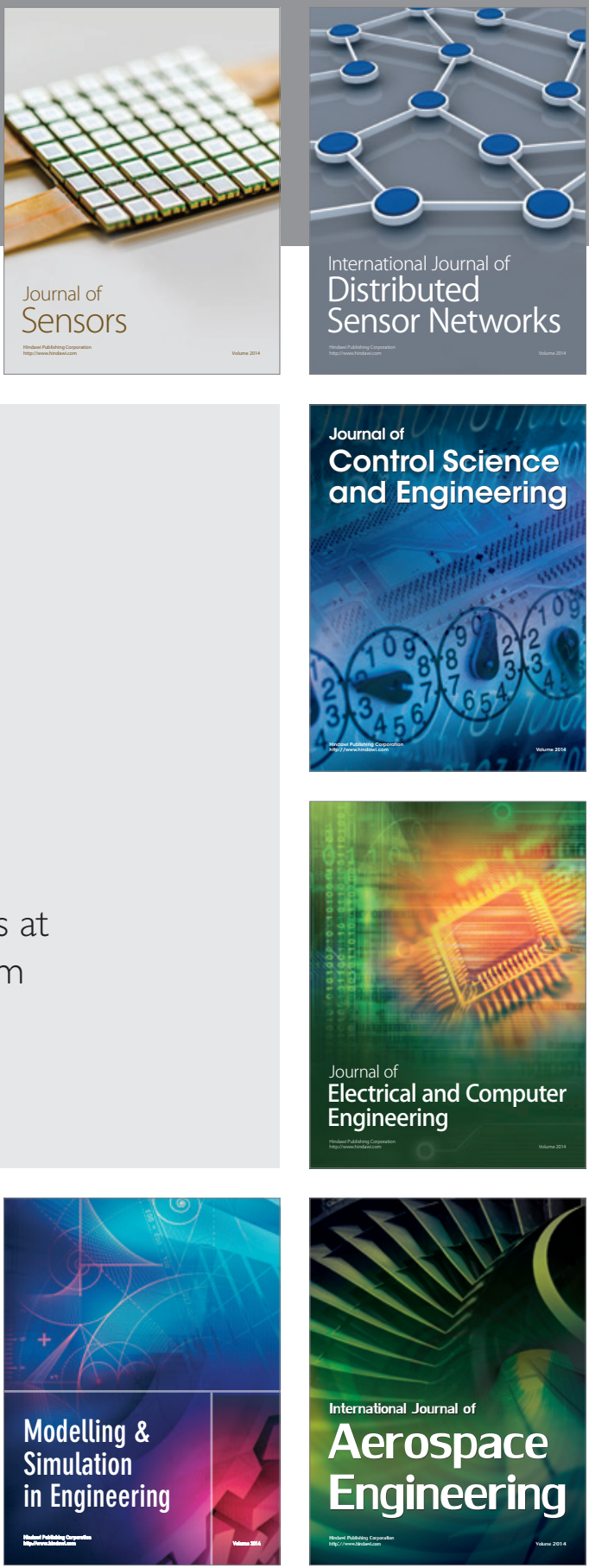

Journal of

Control Science

and Engineering
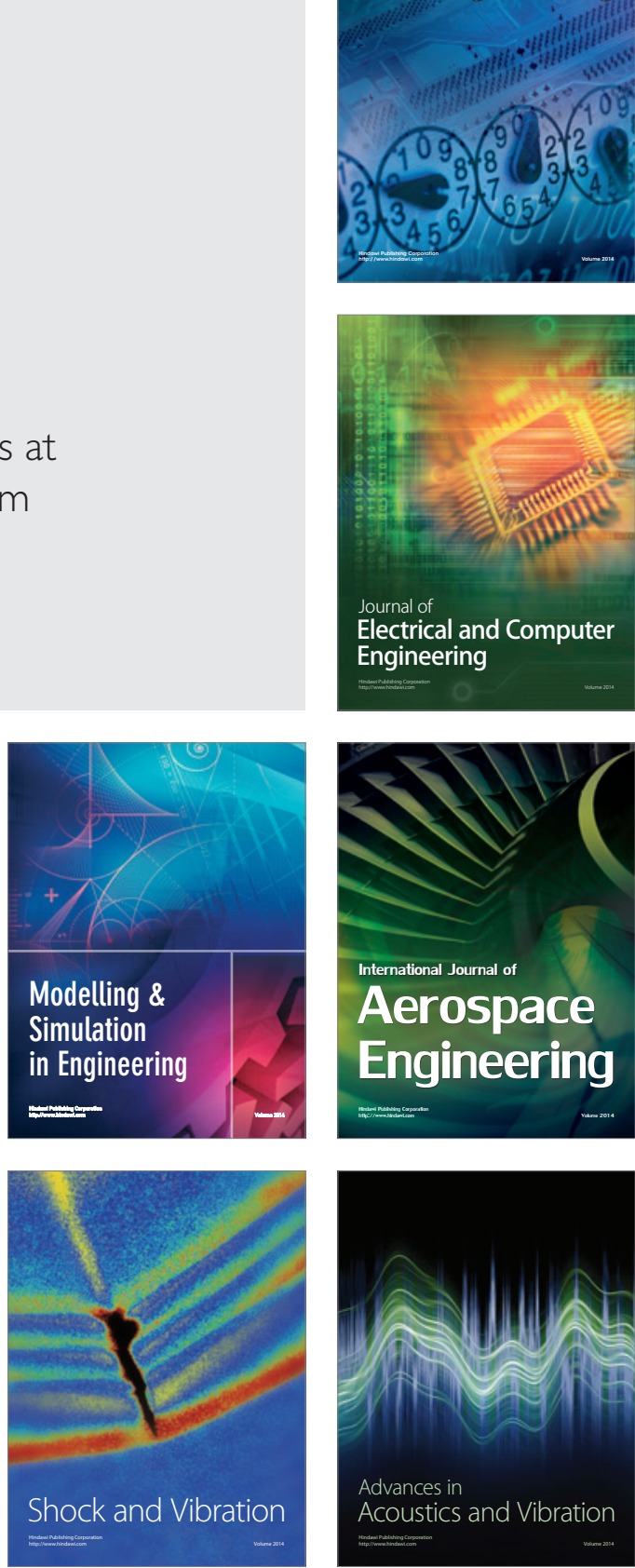